\section{Second Normal Form (2NF)}

Marcelo Arenas

Pontifical Catholic University of Chile, Santiago, Chile

\section{Synonyms}

$2 \mathrm{NF}$

\section{Definition}

Let $R\left(A_{1}, \ldots, A_{n}\right)$ be a relation schema and $\Sigma$ a set of functional dependencies over $R\left(A_{1}, \ldots, A_{n}\right)$. An attribute $A_{i}(i \in\{1, \ldots, n\})$ is a prime attribute if $A_{i}$ is an element of some key of $R\left(A_{1}, \ldots, A_{n}\right)$. Then specification $(R, \Sigma)$ is said to be in second normal form (2NF) if for every nontrivial functional dependency $X \rightarrow A$ implied by $\Sigma$, it holds that $A$ is a prime attribute or $X$ is not a proper subset of any (candidate) key for $R$ [1].

\section{Key Points}

In order to avoid update anomalies in database schemas containing functional dependencies, 2NF was introduced by Codd in [1]. This normal form is defined in terms of the notions of prime attribute and key as shown above. For example, given a relation schema $R(A, B, C)$ and a set of functional dependencies $\Sigma=\{A \rightarrow B\}$, it does not hold that $(R(A, B, C), \Sigma)$ is in $2 \mathrm{NF}$ since $B$ is not a prime attribute and $A$ is a proper subset of the key $A C$. On the other hand, $(S(A, B, C), \Gamma)$ is in 2NF if $\Gamma=\{A \rightarrow B, B \rightarrow C\}$, since $A$ is a key (and thus it is not a proper subset of any candidate key) and $B$ is not contained in any (candidate) key for $S$.

It should be noticed that relation schema $S(A$, $B, C)$ above is in 2NF if $\Gamma=\{A \rightarrow B, B \rightarrow C\}$, although this schema is not in $3 \mathrm{NF}$. In fact, $3 \mathrm{NF}$ is strictly stronger than $2 \mathrm{NF}$; every schema in $3 \mathrm{NF}$ is in $2 \mathrm{NF}$, but there exist schemas (as the one shown above) that are in $2 \mathrm{NF}$ but not in $3 \mathrm{NF}$.

\section{Cross-References}

- Boyce-Codd Normal Form

- Fourth Normal Form

> Normal Forms and Normalization

- Third Normal Form

\section{Recommended Reading}

1. Further CEF. Normalization of the data base relational model. In: Data base systems. Englewood Cliffs: Prentice-Hall; 1972. p. 33-64. 